\title{
El componente P300 como correlato neurofisiológico de la memoria de trabajo conductual en adolescentes con trastorno por déficit de atención/hiperactividad
}

\author{
Patricia Roca, M. Jesús Presentación-Herrero, Ana Miranda-Casas, Fernando Mulas, Pedro Ortiz-Sánchez
}

Introducción. El déficit en funciones ejecutivas ha sido ampliamente estudiado en el trastorno por déficit de atención/ hiperactividad (TDAH). El interés por sus correlatos neurofisiológicos apunta a una mejora en la identificación de los síntomas, las características y los tratamientos para el trastorno.

Objetivo. Analizar, en una muestra de adolescentes con TDAH, la correlación entre la latencia y amplitud del componente P300 y una medida ecológica de memoria de trabajo.

Pacientes y métodos. La muestra estuvo compuesta por 24 chicos y 7 chicas de 12 a 18 años. Se realizó un registro y análisis de potenciales evocados cognitivos P300 en modalidad auditiva en Cz. Además, los padres cumplimentaron una escala de comportamiento ejecutivo en el hogar (BRIEF), de la que se extrajo el índice de memoria de trabajo.

Resultados. Se hallaron correlaciones significativas entre la amplitud del P300 y el índice de memoria de trabajo del BRIEF-P. Conclusión. Los resultados enfatizan la utilidad del componente P300 para el estudio de sus correlatos ejecutivos, y la necesidad de ampliar con estudios que aporten mayores muestras y mayor complejidad.

Palabras clave. Memoria de trabajo. Potenciales evocados. TDAH.

\section{Introducción}

El trastorno por déficit de atención/hiperactividad (TDAH) es un trastorno del neurodesarrollo caracterizado por síntomas de inatención, impulsividad o hiperactividad, que a menudo persiste en la adolescencia e incluso hasta la edad adulta [1,2].

En el intento de describir y explicar la heterogeneidad de este trastorno y sus características han surgido varios modelos teóricos y empíricos que tratan de abarcar el amplio espectro de manifestaciones cognitivas, conductuales y emocionales que se encuentran en las personas con TDAH. Desde el ámbito de la neuropsicología, las funciones ejecutivas han sido objeto de numerosas investigaciones al respecto del TDAH, y se cree que están en la base de parte de las alteraciones funcionales características del trastorno $[3,4]$.

Un trabajo reciente de Lin et al [5] evaluaba el déficit ejecutivo en 300 adolescentes con TDAH, 109 adolescentes con TDAH subclínico, 26 adolescentes con un TDAH que había remitido desde la infancia y un grupo de 263 adolescentes con desarollo típico sin síntomas de TDAH. En el estudio, se identificaron déficits en memoria de trabajo espacial, planificación espacial y memoria de trabajo verbal en el grupo con TDAH y en el grupo subclínico. Tras controlar aspectos como el cociente intelectual, en los grupos de TDAH y TDAH subclínico permanecían alteradas la memoria de trabajo espacial y la planificación espacial.

Un estudio de Fair et al [6] mostró diferencias entre niños con TDAH y controles en un rango de factores cognitivos (inhibición, memoria de trabajo, memoria retentiva, arousal/activación, variabilidad de respuestas, procesamiento temporal y velocidad de procesamiento) y detectó que el grupo de niños con TDAH mostraba una variabilidad similar a la encontrada en los controles. En este estudio, sin embargo, se incluyeron sujetos que habían recibido o estaban recibiendo medicación psicoestimulante (salvo el período de lavado de 24-48 horas), por lo que no se puede descartar que la medicación estimulante pudiera haber tenido efectos sobre el rendimiento neuropsicológico.

Sin embargo, algunos trabajos han sugerido que, al controlar el cociente intelectual (CI) y las tareas que no implican funcionamiento ejecutivo específicamente, desaparecería la disfunción ejecutiva en el TDAH [7]. Por ejemplo, Kuntsi et al emplearon tres tareas neuropsicológicas diferentes que medían respuestas de inhibición, memoria de trabajo y aver-
Departamento de Psicología Evolutiva, Educativa, Social y Metodología; Universitat Jaume I; Castellón (M.J. PresentaciónHerrero). Instituto Valenciano de Neurología Pediátrica, INVANEP (P. Roca, F. Mulas, P. Ortiz-Sánchez) Departamento de Psicología Evolutiva y de la Educación; Universitat de València (A. MirandaCasas). Valencia, España.

Correspondencia:

Dra. Patricia Roca Rodríguez. Instituto Valenciano de Neurología Pediátrica (INVANEP). Artes Gráficas, 23, bajo. E-46010 Valencia.

E-mail:

patricia.roca@invanep.com

Declaración de intereses: Los autores manifiestan la inexistencia de conflictos de interés en relación con este artículo.

Aceptado tras revisión externa: 10.01.14.

Cómo citar este artículo:

Roca P, Presentación-Herrero MJ Miranda-Casas A, Mulas F, OrtizSánchez P. El componente P300 como correlato neurofisiológico de la memoria de trabajo conductual en adolescentes con trastorno por déficit de atención/hiperactividad. Rev Neurol 2014; 58 (Supl 1): S51-6.

(c) 2014 Revista de Neurología 
sión a la demora en una muestra de niños hiperactivos y encontraron que los hiperactivos mostraron un rendimiento peor que el grupo control en la medida de aversión a la demora y en algunos aspectos de las tareas de memoria de trabajo, pero al controlar el efecto del cociente intelectual se disiparon las diferencias en memoria de trabajo [8].

En estudios con grupos adolescentes, se ha afirmado que la disfunción ejecutiva sería también más marcada en adolescentes con TDAH comparados con adolescentes de desarrollo típico, independientemente de edad, sexo, CI global y comorbilidades psiquiátricas, según autores [9-11]. Muchos adolescentes con TDAH presentan déficits en varias áreas del funcionamiento ejecutivo como la memoria de trabajo, el control inhibitorio, la planificación y la atención sostenida [12-15], que se han asociado a un peor rendimiento académico y un peor ajuste social [16-18]. Más en concreto, la memoria de trabajo evaluada a través del Behavior Rating Inventory of Executive Function para padres (BRIEF-P) ha mostrado altas correlaciones, junto con otras funciones ejecutivas, con el funcionamiento académico y el rendimiento escolar de adolescentes con TDAH [15].

La memoria de trabajo se ha propuesto como un déficit nuclear en el TDAH [19]. Se ha definido como un almacén de memoria que opera a corto plazo y que permite al sujeto tener la información disponible el tiempo necesario para procesarla y utilizarla. Mientras que el simple almacenaje de información correspondería a la memoria a corto plazo o span, la memoria de trabajo entendida desde la perspectiva del funcionamiento ejecutivo central implicaría el uso de esa información procesada mentalmente para guiar el comportamiento hacia una meta $[20,21]$ y se identificarían tres aspectos dentro de la memoria de trabajo: procesos de actualización continua, procesamiento dual y reordenamiento serial (o control del orden temporal).

Algunos estudios han acotado varios aspectos de la memoria de trabajo y han evaluado de manera separada tareas de memoria de trabajo verbal o visuoespacial; así, se ha encontrado una relación con los síntomas de inatención $[18,22,23]$ e impulsividad [24]. Otro trabajo reciente de Coghill et al [25] exploraba el rendimiento neuropsicológico de 83 niños con TDAH y 66 controles en seis dominios de funcionamiento neuropsicológico: memoria de trabajo, inhibición, aversión a la demora, toma de decisiones, timing y variabilidad de respuesta. Para evaluar la memoria de trabajo, estos autores emplearon las tareas Delayed Matching to Sample (DMS) y Spatial Working Memory (SWM). Encontraron que el grupo TDAH presentaba una ejecución peor en todos los dominios y eran mayores los tamaños del efecto para las medidas de aversión a la demora $(0,82)$ y memoria $(0,95)$.

De hecho, algunos estudios han apuntado que, en el subgrupo de TDAH con presentación puramente inatenta, la asociación de los síntomas de inatención con el déficit de memoria de trabajo superaría a la relación ya establecida con el déficit inhibitorio [12,26]. Los estudios apuntan a que el déficit en memoria de trabajo se asociaría a mayores problemas de aprendizaje y menor rendimiento académico, tanto en niños con TDAH como sin el trastorno [27-28].

Sin embargo, sobre este déficit en memoria de trabajo existen resultados inconsistentes. Los metaanálisis demuestran que los valores de afectación de la memoria de trabajo varían en función del tipo de tarea planteada, lo que dificulta la extracción de conclusiones acerca de esta relación, puesto que la tarea planteada y el paradigma empleado varía de unos estudios a otros [29-30].

A pesar de los numerosos estudios sobre las funciones ejecutivas en el TDAH, sigue sin identificarse claramente un patrón ejecutivo concreto que dé cuenta de las manifestaciones cognitivas y conductuales del trastorno [31-32].

Es más, entre las críticas dirigidas a estos estudios, se ha señalado que los instrumentos neuropsicológicos empleados podrían estar midiendo no sólo el componente ejecutivo que es objeto de medición, que correspondería al resultado final de la tarea, sino además otras destrezas no ejecutivas que contribuyen al funcionamiento de dicho componente (como el procesamiento espacial, destrezas motoras o perceptivas) y resultan a menudo obviadas $[5,12]$.

Además, los tests neuropsicológicos de laboratorio han sido criticados por su baja validez ecológica, aduciendo que estos tests no serían adecuados para medir y explicar la capacidad del sujeto para organizar el comportamiento y planificar un plan de acción dirigido a conseguir metas a largo plazo y en entornos de la vida real. Por lo tanto, se ha sugerido que estos tests tendrían menor poder predictivo de alteraciones en la vida diaria que otro tipo de medidas $[4,33]$. Un trabajo reciente de Toplak et al revisaba esta cuestión y señalaba una baja correlación entre la puntuación en escalas de funcionamiento ejecutivo y el rendimiento en tareas neuropsicológicas de laboratorio [34].

Otro intento por analizar las diferentes manifestaciones cognitivas y conductuales en el TDAH procede de la neurofisiología. En esta área, los potenciales evocados cognitivos han sido tradicional- 
mente asociados a varios aspectos del funcionamiento cognitivo, emocional y comportamental en el TDAH [35,36]. El componente evocado cognitivo P300 ha sido sin duda el que mayor apoyo empírico ha recibido y destacan las aportaciones en el estudio de varios aspectos del funcionamiento ejecutivo como el control inhibitorio, el control de impulsos y la memoria de trabajo, entre otros [37-41].

Mientras que la latencia de la onda P300 se ha asociado a la velocidad de procesamiento y la dificultad para clasificar estímulos [42], numerosos estudios con varios paradigmas han relacionado una menor amplitud del componente P300 con peor rendimiento y mayores dificultades de atención selectiva, control inhibitorio, control de interferencia, distintos tipos de memoria y procesamiento emocional [43].

En trabajos anteriores, nuestro equipo de trabajo ha encontrado correlaciones significativas entre el componente P300 y distintas medidas de funcionamiento ejecutivo en niños con TDAH [41]. El objetivo de este trabajo es explorar la relación existente entre una medida ecológica de memoria de trabajo (medida a través de la subescala del BRIEF) y los valores de latencia y amplitud del componente P300$\mathrm{Cz}$ en un grupo de adolescentes con TDAH.

\section{Pacientes y métodos}

\section{Muestra}

La muestra estuvo compuesta por 31 chicos y chicas de 12-18 años, el 77,4\% $(n=24)$ de los cuales eran chicos y el $22,6 \%$, chicas $(n=7)$. De todos ellos, 11 tenían un diagnóstico de TDAH subtipo combinado $(36,7 \%)$ y 20 , de TDAH subtipo inatento (63,3\%). Todos eran pacientes naïve que nunca habían tomado tratamiento farmacológico para el TDAH.

\section{Instrumentos y procedimiento}

Para evaluar la memoria de trabajo conductual, se solicitó a los padres que completaran el BRIEF-P [44]. El BRIEF ha mostrado su utilidad en el perfil diagnóstico y ejecutivo en niños y adolescentes con TDAH [45]. Contiene 86 ítems y consta de ocho escalas empíricas (inhibición, cambio de set, control emocional, iniciativa, memoria de trabajo, planificación, orden de materiales y control) y dos escalas globales (el índice de regulación del comportamiento y el índice de metacognición). La escala de memoria de trabajo explora la capacidad para mantener información en la mente con el objeto de com- pletar una tarea, registrar y almacenar información o generar objetivos. Algunos ítems que forman parte de esta escala son 'Cuando se le asignan tres cosas para hacer, sólo recuerda la primera o la última de ellas', 'Necesita ayuda de los adultos para permanecer en la tarea,' 'Cuando se le envía a traer algo, se olvida de lo que se supone que debería traer, entre otros. La escala puede puntuar entre 0 y 30 . Las puntuaciones directas reflejan el grado de dificultad que presenta el niño en el área evaluada. Las puntuaciones más altas indican mayor grado de disfunción ejecutiva.

El registro y análisis de potenciales evocados se realizó mediante un paradigma oddball en modalidad auditiva, siguiendo las recomendaciones de Duncan et al [46].

Para el registro de los potenciales, se sentó al sujeto en una sala aislada de ruidos, en penumbra y con los ojos cerrados. Se presentaron 200 estímulos auditivos de 70 dB y 100 ms de duración, con un intervalo de $1 \mathrm{~s}$ entre estímulos, con frecuencias de $500 \mathrm{~Hz}$ (estímulos no diana) y $1.000 \mathrm{~Hz}$ (estímulos diana), con un orden aleatorio de aparición de los estímulos con una proporción $80 \%-20 \%$ respectivamente, evitando el solapamiento de estímulos diana. Se realizó un ensayo previo de ajuste, y se daba al sujeto la instrucción de apretar un botón cuando escuchara los estímulos diana. El registro de la respuesta eléctrica cerebral se realizó con electrodos de superficie convencionales de cloruro de plata, colocando uno sobre la localización $\mathrm{Cz}$, con referencia en los lóbulos de las orejas y tierra en $\mathrm{AFz}, \mathrm{y}$ con impedancias $\leq 5 \mathrm{k} \Omega$. La señal se registró mediante el sistema digital Cognitrace-Eemagine ANT, sincronizado con el estimulador Eevoke.

Para el análisis del componente P300, se empleó el sistema Eemagine. Se aplicaron filtros de 0,01$100 \mathrm{~Hz}$ y se eliminaron segmentos artefactados. Se analizaron las ondas promedio de los estímulos diana y no diana por separado para cada electrodo y para cada modalidad, con una ventana de análisis de 280-450 ms. Se localizó el pico de mayor amplitud de la onda positiva P3 y se estableció el valor en microvoltios. Se estableció el valor de latencia en milisegundos a partir del momento inicial de aparición del estímulo.

\section{Resultados}

Para analizar la relación en el grupo TDAH entre los datos del componente $\mathrm{P} 300$ en $\mathrm{Cz}$ y los resultados obtenidos en la medida de memoria de trabajo, se realizaron correlaciones bivariadas. Todos los aná- 
Tabla I. Prueba $t$ de Student para muestras independientes de la comparación de medias entre el cluster de menor edad y el de mayor edad, en la medida de memoria de trabajo del BRIEF y los valores de latencia y amplitud del P300 en CZ.

\begin{tabular}{|c|c|c|c|c|c|c|}
\hline & \multicolumn{2}{|c|}{ Cluster 1 (edad media: 12) } & \multicolumn{3}{|c|}{ Cluster 2 (edad media: 17,7) } & \multirow{2}{*}{$p$} \\
\hline & $x$ & $\delta$ & $x$ & $\delta$ & $t$ & \\
\hline $\begin{array}{l}\text { Memoria de } \\
\text { trabajo BRIEF-P }\end{array}$ & 24,77 & 3,05 & 21,16 & 3,31 & 0,009 & 0,045 \\
\hline Latencia P300-Cz & 338,53 & 28,55 & 330,50 & 42,16 & 1,950 & 0,675 \\
\hline Amplitud P300-Cz & 10,25 & 3,55 & 11,74 & 2,39 & 0,861 & 0,253 \\
\hline
\end{tabular}

Tabla II. Correlaciones bivariadas entre variables del P300 (latencia y amplitud) en modalidad auditiva y el índice de memoria de trabajo del BRIEF-P, en los clusters por edad.

\begin{tabular}{|c|c|c|c|c|}
\hline & \multicolumn{2}{|c|}{ Cluster 1 (edad media: 12) } & \multicolumn{2}{|c|}{ Cluster 2 (edad media: 17,7 ) } \\
\hline & Latencia & Amplitud & Latencia & Amplitud \\
\hline & $\mathrm{P} 300-\mathrm{Cz}$ & $\mathrm{P} 300-\mathrm{Cz}$ & $\mathrm{P} 300-\mathrm{CZ}$ & P300-Cz \\
\hline $\begin{array}{l}\text { Memoria de } \\
\text { trabajo BRIEF-P }\end{array}$ & 0,094 & $-0,480^{a}$ & 0,605 & $-0,387$ \\
\hline
\end{tabular}

lisis estadísticos se llevaron a cabo con la versión 17.0 del programa SPSS.

Se comprobó que no había diferencias en las variables empleadas (latencia del P300, amplitud del P300 e índice de memoria de trabajo del BRIEF-P) entre chicos y chicas, y tampoco hubo diferencias entre los subtipos.

La correlación entre la latencia del P300 auditivo en $\mathrm{Cz}$ y el índice de memoria de trabajo del BRIEF-P no resultó estadísticamente significativa $(r=0,135)$. Por otro lado, la correlación entre la amplitud del P300 en Cz y el índice de memoria de trabajo resultó estadísticamente significativa al nivel 0,01 $(r=$ $-0,487)$. Estos resultados apuntan a que una mayor amplitud del componente $\mathrm{P} 300$ en Cz vendría asociada a menores dificultades en la medida de memoria de trabajo conductual.

Al hilo de estos resultados, se calcularon dos clusters por el procedimiento de $k$ medias con dos iteraciones, que dividían a la muestra por edad; el primero de ellos agrupaba a 25 sujetos con una media de 12 años y el segundo cluster agrupó a 6 sujetos con una edad media de 17,17 años. Mientras que los cluster formados no mostraron diferencias significativas en cuanto a la latencia y amplitud del
P300 (Tabla I), sí se encontraron diferencias significativas en el índice de memoria de trabajo del BRIEF-P, que mostraron que el grupo de sujetos más pequeños tiene más dificultades en memoria de trabajo conductual informada por los padres, en comparación con el grupo de sujetos mayores.

Al repetir el análisis de correlaciones dividiendo los sujetos por su pertenencia a uno u otro cluster de edad (Tabla II), los resultados sufrieron una variación: aunque la dirección de las correlaciones se mantenía (la amplitud del componente P300 mostraba una correlación negativa con el índice de memoria de trabajo), sólo en el grupo de sujetos más pequeños se mantuvo la significación. Es decir, en nuestra muestra, una mayor amplitud del componente P300 en Cz vendría asociada a menores dificultades en una medida de memoria de trabajo conductual en los niños más pequeños, pero no así en los mayores.

\section{Discusión}

Nuestros resultados han mostrado una correlación negativa entre la amplitud del P300 y el índice de memoria de trabajo del BRIEF-P. Esta correlación significaría que una mayor amplitud del P300 auditivo en $\mathrm{Cz}$ se asociaría a menores dificultades de memoria de trabajo en la vida diaria. Sin embargo, la separación de los sujetos por mayor y menor edad ha mostrado que las dificultades de memoria de trabajo reflejadas en esta escala conductual eran mayores en los sujetos de menor edad, y sólo en estos chicos se mantenía la correlación resaltada a la vez que desaparecía la asociación en los sujetos mayores.

Esta correlación iría en la línea de los descrito en trabajos anteriores acerca de que una menor amplitud del componente P300 estaría asociada a un peor rendimiento en memoria de trabajo, tanto en los estudios que empleaban medidas ecológicas como el BRIEF como los que empleaban otras medidas de laboratorio de memoria de trabajo $[41,43]$.

Sin embargo, los resultados también resaltan las dificultades en el estudio de la memoria de trabajo en el TDAH, al disiparse la relación entre nuestra medida de memoria de trabajo y el componente P300 en los sujetos de mayor edad, aunque este resultado debe tomarse con cautela debido al escaso tamaño del cluster. Esta dificultad se añade a la idea de que la afectación de la memoria de trabajo variaría en función del tipo de tarea planteada y el instrumento empleado [30].

Este estudio no está libre de limitaciones. En primer lugar, la falta de un grupo de control de sujetos 
con desarrollo típico impide la aplicación de estadística comparativa-inferencial. Los análisis emprendidos únicamente permiten deducir que parece producirse una asociación entre ambos fenómenos, memoria de trabajo y P300, en el grupo de sujetos con TDAH. El presente estudio supone una primera aproximación en esta línea y, sin duda, la inclusión en futuras investigaciones de un grupo control de sujetos con desarrollo típico permitirá un diseño comparativo del cual obtener resultados inferenciales acerca del componente evolutivo del potencial evocado cognitivo, así como de otros correlatos presentes en población general. En segundo lugar, el número total de sujetos es reducido, con una muestra mayor se podrían extraer conclusiones más ricas, dada la demostrada heterogeneidad de resultados en estas poblaciones. En tercer lugar, es necesario destacar que contamos con pocos sujetos de edades más elevadas y la mayoría de sujetos son de menor edad. Quizá con mayor número de sujetos de más edad se encontrarían relaciones más potentes entre las variables medidas. Por último, para este trabajo inicial se ha seleccionado una medida de memoria de trabajo de corte ecológico. Sería interesante en trabajos futuros añadir diferentes medidas de laboratorio de memoria de trabajo verbal y visuoespacial, estáticas y dinámicas, con distintas tareas y modalidades de estímulo. Estas diferentes medidas de memoria aumentarían sin duda la precisión de los resultados.

En este trabajo, se ha tratado de superar la limitación de los tests de laboratorio con una medida más ecológica de memoria de trabajo, el BRIEF-P. Tiene también un valor orientativo para la práctica de futuras investigaciones en esta línea al apuntar una relación significativa entre la amplitud del componente P300 y menores dificultades en memoria de trabajo en adolescentes con TDAH, especialmente en los de menor edad. Son necesarios futuros estudios que desarrollen estos aspectos.

\section{Bibliografía}

1. Barkley RA, Fischer M, Smallish L, Fletcher K. Young adult outcome of hyperactive children: adaptive functioning in major life activities. J Am Acad Child Adolesc Psychiatry 2006; 45: 192-202.

2. American Psychiatric Association. Manual diagnóstico y estadístico de los trastornos mentales, cuarta edición, texto revisado. Barcelona: Masson; 2002.

3. Barkley RA. The executive functions and self-regulation: an evolutionary neuropsychological perspective. Neuropsychol Rev 2001; 11: 1-29.

4. Barkley RA, Fischer M. Predicting impairment in major life activities and occupational functioning in hyperactive children as adults: self-reported executive function (EF) deficits vs. $\mathrm{EF}$ tests. Dev Neuropsychol 2011; 36: 137-61.

5. Lin YJ, Chen WJ, Gau SS. Neuropsychological functions among adolescents with persistent, subsyndromal and remitted attention deficit hyperactivity disorder. Psychol Med 2013; 27: 1-13.

6. Fair DA, Bathula D, Nikolas MA, Nigg JT. Distinct neuropsychological subgroups in typically developing youth inform heterogeneity in children with ADHD. Proc Natl Acad Sci U S A 2012; 109: 6769-74.

7. Scheres A, Oosterlaan J, Geurts H, Morein-Zamir S, Meiran N, Schut $\mathrm{H}$, et al. Executive functioning in boys with ADHD: primarily an inhibition deficit? Arch Clin Neuropsychol 2004; 19: 569-94.

8. Kuntsi J, Oosterlaan J, Stevenson J. Psychological mechanisms in hyperactivity: I. Response inhibition deficit, working memory impairment, delay aversion, or something else? J Child Psychol Psychiatry 2001; 42: 199-210.

9. Seidman LJ, Biederman J, Monuteaux MC, Valera E, Doyle AE, Faraone SV. Impact of gender and age on executive functioning: do girls and boys with and without attention deficit hyperactivity disorder differ neuropsychologically in preteen and teenage years? Dev Neuropsychol 2005; 27: 79-105.

10. Martel M, Nikolas M, Nigg JT. Executive function in adolescents with ADHD. J Am Acad Child Adolesc Psychiatry 2007; 46: 1437-44.

11. Gau SS, Chiu CD, Shang CY, Cheng AT, Soong WT. Executive function in adolescence among children with attention-deficit/ hyperactivity disorder in Taiwan. J Dev Behav Pediatr 2009; 30: 525-34.

12. Willcutt EG, Doyle AE, Nigg JT, Faraone SV, Pennington BF. Validity of the executive function theory of attentiondeficit/hyperactivity disorder: a meta-analytic review. Biol Psychiatry 2005; 57: 1336-46.

13. Hinshaw SP, Carte ET, Fan C, Jassy JS, Owens EB. Neuropsychological functioning of girls with attention-deficit/ hyperactivity disorder followed prospectively into adolescence: evidence for continuing deficits? Neuropsychology 2007; 21: 263-73.

14. Thorell LB. Do delay aversion and executive function deficits make distinct contributions to the functional impact of ADHD symptoms? A study of early academic skill deficits. J Child Psychol Psychiatry 2007; 48: 1061-70.

15. Langberg JM, Dvorsky MR, Evans SW. What specific facets of executive function are associated with academic functioning in youth with attention-deficit/hyperactivity disorder? J Abnorm Child Psychol 2013; 41: 1145-59.

16. Gropper RJ, Tannock R. A pilot study of working memory and academic achievement in college students with ADHD. J Atten Dis 2009; 12: 574-81.

17. Rogers M, Hwang H, Toplak M, Weiss M, Tannock R. Inattention, working memory, and academic achievement in adolescents referred for attention deficit/hyperactivity disorder (ADHD). Child Neuropsychol 2011; 17: 444-58.

18. Kofler MJ, Rapport MD, Bolden J, Sarver DE, Raiker JS, Alderson RM. Working memory deficits and social problems in children with ADHD. J Abnorm Child Psychol 2011; 39: 805-17.

19. Rapport MD, Chung KM, Shore G, Isaacs P. A conceptual model of child psychopathology: Implications for understanding attention deficit hyperactivity disorder and treatment efficacy. J Clin Child Psychol 2001; 30: 48-58.

20. Baddeley A. Working memory. Curr Biol 2010; 20: 136-40.

21. Cowan N. The focus of attention as observed in visual working memory tasks: making sense of competing claims. Neuropsychologia 2011; 49: 1401-6.

22. Takeuchi A, Ogino T, Hanafusa K, Morooka T, Oka M, Yorifuji $\mathrm{T}$, et al. Inhibitory function and working memory in attention deficit/hyperactivity disorder and pervasive developmental disorders: does a continuous cognitive gradient explain ADHD and PDD traits? Acta Med Okayama 2013; 67: 293-303.

23. Burgess GC, Depue BE, Ruzic L, Willcutt EG, Du YP Banich MT. Attentional control activation relates to working memory in attention-deficit/hyperactivity disorder. Biol Psychiatry 2010; 67: 632-40. 
24. Raiker JS, Rapport MD, Kofler MJ, Sarver DE. Objectivelymeasured impulsivity and attention-deficit/hyperactivity disorder (ADHD): testing competing predictions from the working memory and behavioral inhibition models of ADHD. J Abnorm Child Psychol 2012; 40: 699-713.

25. Coghill DR, Seth S, Matthews K. A comprehensive assessment of memory, delay aversion, timing, inhibition, decision making and variability in attention deficit/hyperactivity disorder: advancing beyond the three-pathway models. Psychol Med 2013; 31: 1-13.

26. Kofler MJ, Rapport MD, Bolden J, Sarver DE, Rainker JS. ADHD and working memory: the impact of central executive deficits and exceeding storage/rehearsal capacity on observed inattentive behavior. J Abnorm Child Psychol 2010; 38: 149-61.

27. Alloway TP, Alloway RG. Investigating the predictive roles of working memory and IQ in academic attainment. J Exp Child Psychol 2010; 106: 20-9.

28. Alloway TP, Elliott J, Place M. Investigating the relationship between attention and working memory in clinical and community samples. Child Neuropsychol 2010; 16: 242-54.

29. Barkley RA, Edwards G, Laneri M, Fletcher K, Metevia L. Executive functioning, temporal discounting, and sense of time in adolescents with attention deficit hyperactivity disorder (ADHD) and oppositional defiant disorder (ODD). J Abnorm Child Psychol 2001; 29: 541-56.

30. Martinussen R, Hayden J, Hogg-Johnsen S, Tannock R. A meta-analysis of working memory in children with attention-deficit/hyperactivity disorder. J Am Acad Child Adolesc Psychiatry 2005; 44: 377-84.

31. Doyle AE. Executive functions in attention-deficit/ hyperactivity disorder. J Clin Psychol 2006; 67 (Suppl 8): 21-6.

32. Wodka EL, Mostofsky SH, Prahme C, Gidley Larson IC, Loftis C, Denckla MB, et al. Process examination of executive function in ADHD: sex and subtype effects. Clin Neuropsychol 2008; 22: 826-41.

33. Barkley RA, Murphy KR. The nature of executive function (EF) deficits in daily life activities in adults with ADHD and their relationship to performance on EF tests. J Psychopathol Behav 2010; 33: 137-58.

34. Toplak ME, West RF, Stanovich KE. Practitioner review: do performance-based measures and ratings of executive function assess the same construct? J Child Psychol Psychiatry 2013; 54: 131-43.
35. Chiapa $\mathrm{KH}$, ed. Evoked potentials in clinical medicine. New York: Raven Press; 1989.

36. Banachewski T, Brandeis D. Annotation: what electrical brain activity tells us about brain function that other techniques cannot tell us - a child psychiatric perspective. J Child Psychol Psychiatry 2007; 48, 5: 415-35.

37. Kutas M, McCarthy G, Donchin E. Augmenting mental chronometry: the P300 as a measure of stimulus evaluation time. Science 1977; (4305): 792-5.

38. Magliero A, Bashore TR, Coles MG, Donchin E. On the dependence of P300 latency on stimulus evaluation processes. Psychophysiology 1984; 21: 171-86.

39. Doenhert M, Brandeis D, Imhof K, Drechsler R, Steinhausen HC. Mapping attention-deficit/hyperactivity disorder from childhood to adolescence - no neurophysiologic evidence for a developmental lag of attention but some for inhibition. Biol Psychiatry 2010; 67: 608-16.

40. Senderecka M, Grabowska A, Szewczyk J, Gerc K, Chmylak R. Response inhibition of children with ADHD in the stop-signal task: an event-related potential study. Int J Psychophysiol 2012; 85: 93-105.

41. Roca P, Mulas F, Presentación MJ, Ortiz P, Idiazábal MA, Miranda A. Potenciales evocados y funcionamiento ejecutivo en niños con trastorno por déficit de atención/hiperactividad. Rev Neurol 2012; 54 (Supl 1): S95-103.

42. Picton TW. The P300 wave of the human event related potential J Clin Neurophysiol 1992; 9: 4567-9.

43. Johnstone SJ, Barry RJ, Clarke AR. Ten years on: a follow-up review of ERP research in attention-deficit/hyperactivity disorder. Clin Neurophysiol 2012; 124: 644-57.

44. Gioia G, Isquith P, Guy S, Kenworthy L. The Behavior Rating Inventory of Executive Function. Lutz, FL: Psychological Assessment Resources; 2000

45. McCandless S, O'Laughlin L. The clinical utility of the Behavior Rating Inventory of Executive Function (BRIEF) in the diagnosis of ADHD. J Atten Disord 2007; 10: 381-9.

46. Duncan CC, Barry RJ, Connolly JF, Fischer C, Michie PT, Näätänen R, et al. Event-related potentials in clinical research guidelines for eliciting, recording, and quantifying mismatch negativity, P300, and N400. Clin Neurophysiol 2009; 120: 1883-908.

\section{The P300 component as a neurophysiological correlate of behavioural working memory in adolescents with attention deficit hyperactivity disorder}

Introduction. Deficit in the executive functions has been widely studied in attention deficit hyperactivity disorder (ADHD) The interest in its neurophysiological correlates has led to an improvement in the identification of the symptoms, the characteristics and the treatment of the disorder.

Aims. The objective of this study was to analyse the correlation between latency and amplitude of the P300 component and an ecological measure of working memory in a sample of adolescents.

Patients and methods. The sample consisted of 24 boys and 7 girls aged between 12 and 18 years. P300 cognitive evoked potentials in auditory mode at $\mathrm{Cz}$ were registered and analysed. Furthermore, the parents filled in an executive behaviour home rating scale (BRIEF), which the working memory index was extracted from.

Results. Significant correlations were found between the P300 amplitude and the working memory index of the BRIEF-P.

Conclusions. Results underline the usefulness of the P300 component for the study of its executive correlates, as well as the need to conduct further studies involving larger samples and greater complexity.

Key words. ADHD. Evoked potentials. Working memory. 